\title{
Viscosity solutions for elliptic-parabolic problems
}

\author{
Paola MANNUCCI \\ Dipartimento di Matematica Pura e Applicata \\ Università degli Studi di Padova \\ Via Belzoni 7 \\ 35131 Padova, Italy \\ e-mail: mannucci@math.unipd.it \\ Juan Luis VAZQUEZ \\ Departamento de Matemáticas \\ Universidad Autónoma de Madrid \\ Campus de Cantoblanco \\ 28049 Madrid, Spain \\ e-mail: juanluis.vazquez@uam.es
}

\begin{abstract}
We study an elliptic-parabolic problem appearing in the theory of partially saturated flows in the framework of viscosity solutions. This is part of current investigation to understand the theory of viscosity solutions for PDE problems involving free boundaries. We prove that the problem is well posed in the viscosity setting and compare the results with the weak theory. Dirichlet or Neumann boundary conditions are considered.

2000 Mathematics Subject Classification: 35D05, 35K65, 35R35.

Key words: viscosity solution, elliptic-parabolic problems, free boundary problem.
\end{abstract}

\section{Introduction}

In this paper we are interested in the well-posedness of the following ellipticparabolic problem:

$$
\begin{cases}c(u)_{t}=\Delta u, & \text { in } \Omega \times(0, T), \\ c(u)=v_{0}, & \text { on } \Omega \times\{0\}, \\ u=g, & \text { on } \partial \Omega \times(0, T),\end{cases}
$$

where $\Omega$ is an open subdomain of $R^{N}$, and the constitutive function $c(\eta) \in C^{0}(\mathbf{R})$ satisfies $c(\eta) \equiv 0$, if $\eta \leq 0$, while it is a $C^{1}$ strictly increasing function if $\eta>0$. 
(See figure 1)

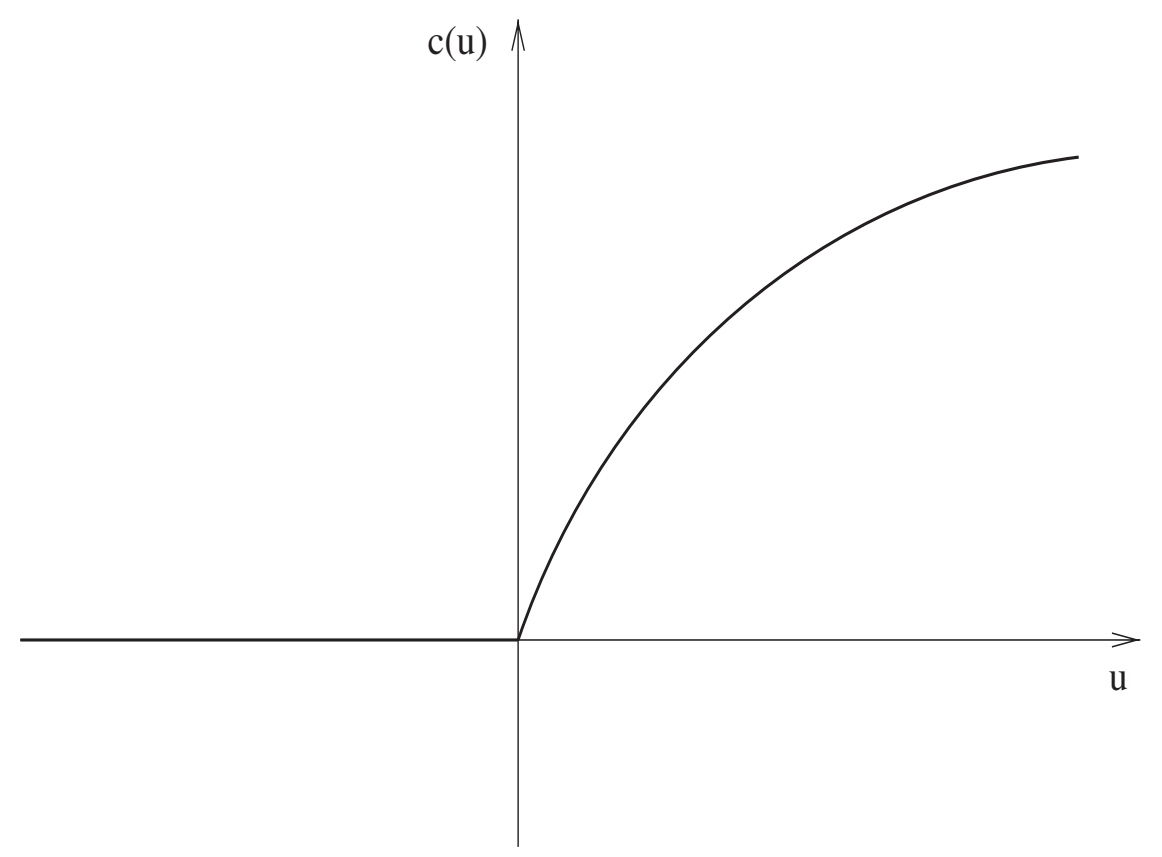

Figure 1

Equation (1.1) is not uniformly parabolic. The level set $\{u=0\}$ splits the problem in two regions in which the equation is respectively parabolic and elliptic.

This type of equation arises in the theory of partially saturated flows in porous media. In this context, $u$ represents the pressure or the hydrostatic potential of the fluid, and $c(u)$ the saturation or moisture content of the medium. In the multidimensional case, the cornerstone is the existence and uniqueness of a weak solution in the paper of Alt - Luckhaus [1]. Further regularity of that solution is obtained by Di Benedetto - Gariepy in [12]. When posed in one space dimension (see [14]), much more is known: a complete analysis of existence, uniqueness and regularity of a weak solution of this problem has been performed by Van Duijn Peletier and Bertsch - Hulshof in several papers (see [13] and [5] and the references therein).

In this paper we study the problem in the setting of viscosity solutions. Indeed, this work can be seen as part of a general program of defining viscosity solutions and of establishing the well-posedness for parabolic problems involving free boundaries. The standard theory on viscosity solutions, introduced by Crandall, Evans, Lions, [11], can not be used in this case. Some basic references on viscosity solutions are [3], [4], [8]. We consider the one-dimensional case where a general theory of regularity of weak solutions and free boundaries is known. 
Our aim is to give a suitable definition of viscosity solution for problem (1.1). This definition allows us to prove that the problem (1.1) is well posed in that setting. In particular, we find a comparison principle between viscosity solutions and weak solutions, thus proving that the viscosity solution in our problem coincides with the weak solution. To do this we use some tools introduced by Caffarelli and Vazquez in [10] where the authors give a suitable definition of a viscosity solution for the porous medium equation:

$$
u_{t}=u \Delta u+|\nabla u|^{2}
$$

See [6] for an extension of that result. These definitions are inspired in earlier work of Caffarelli on stationary free boundary problems, see [7], continued by him and coauthors for the Stefan Problem, cf. [2]. While for Stefan and porous medium equations the motion of the interface is locally determined by the space slope of the solutions near the point, this is not true in our case, a situation that reminds of the combustion problem treated in [9] and complicated the analysis.

Similar definitions of viscosity solution can be used for a large class of problems involving free boundaries. Where a general theory for weak solutions exists, the aim is to prove that the viscosity solution exists, it is unique and it coincides with the weak solution, thus making for a foundation for the viscosity approach. In more general situations, the viscosity theory could be developed without the help of weak solutions.

\section{Problem setting. Classical free boundary solutions}

Let the space domain be an open interval in $\mathbf{R}$. We take, for the sake of simplicity, $I=(0,1)$. We consider the following problem

$$
\begin{array}{lr}
\frac{\partial c(u)}{\partial t}-\frac{\partial^{2} u}{\partial x^{2}}=0 & \text { in } \quad(0,1) \times(0, T) \equiv: Q_{T}, \\
c(u(x, 0))=v_{0}(x) & \text { on }(0,1), \\
u(0, t)=g_{0}(t) & \text { on }(0, T), \\
u(1, t)=g_{1}(t) & \text { on }(0, T),
\end{array}
$$

with the stated assumptions on $c$. This is the problem with Dirichlet boundary conditions, that we will refer as (PD). We make the following assumptions on the data of this problem. On the boundary data we assume that $g_{0}(t), g_{1}(t)$ are continuous functions on $[0, T]$ and

$$
g_{0}(t)>0, \quad g_{1}(t)<0, \forall t \in(0, T),
$$


while the initial data satisfies:

$$
\begin{aligned}
& v_{0}(x) \text { is a nonnegative continuous function } \\
& v_{0}(x)>0 \Longleftrightarrow x \in\left[0, x_{0}\right), x_{0} \in(0,1) .
\end{aligned}
$$

Replacing conditions (2.3) and (2.4) by

$$
u_{x}(0, t)=f_{0}(t), \quad u_{x}(1, t)=f_{1}(t),
$$

we obtain the corresponding Neumann problem, (PN), that will be briefly considered in the last section.

As stated in the Introduction, problems consisting of (2.1)-(2.2) with either Dirichlet or Neumann conditions are well studied from the classical point of view. In the one-dimensional case we have quoted the papers of [13], [5] and their references. Under suitable assumptions on the data, the authors extensively study the regularity of the solution and of the free boundary for the Dirichlet and for the Neumann problem. More precisely, they prove that there exists an unique weak solution $u$ of problem $(2.1)-(2.4)$ such that $c(u) \in C\left(\bar{Q}_{T}\right), u_{x} \in C\left(Q_{T}\right)$, $u_{t} \in L_{\text {loc }}^{\infty}\left(Q_{T}\right)$, where $T$ is a suitable constant determined by the data. It is shown that the solution $u(x, t)$ is smooth and a classical solution of the equation where it does not vanish, but it has limited regularity on the set of points $(x, t)$ where it vanishes.

\subsection{Classical free boundary theory}

Therefore, it will be convenient to introduce the following definitions that will be useful in constructing the theory of viscosity solutions.

Definition 2.1 We say that a function $u(x, t) \in C^{0}\left(Q_{T}\right)$ is a classical freeboundary solution of equation (2.1) if

(i) the set $\Gamma(u)$ where $u=0$ is representable as a function $x=s(t)$ such that $s(t)$ is Lipschitz continuous on $[0, T]$.

(ii) $u$ is $C_{x, t}^{2,1}$ smooth in the positivity set $\mathcal{P}(u)$ where it solves $c^{\prime}(u) u_{t}=u_{x x}$. In the negativity set $\mathcal{N}(u), u$ solves $u_{x x}=0$ and $u_{t} \in L^{\infty}$.

(iii) $u_{x}$ is continuous in $Q_{T}$.

In the set $\mathcal{P}(u)$, called the unsaturated region, equation (2.1) is parabolic; $\mathcal{S}(u)=\{u \leq 0\}$ is the saturated region, inside it equation (2.1) becomes elliptic. The set $\Gamma(u)$ is called the free boundary. Note that the most important information in (iii) is that $u_{x}$ is continuous across the free boundary.

Definition 2.2 We say that a function $u(x, t) \in C^{0}([0,1] \times[0, T))$ is a classical free-boundary solution of problem (PD) if it is a classical free boundary solution of the equation in $Q_{T}$ and moreover,

(iv) $c(u(x, 0))=v_{0}(x)$,

(v) $u(0, t)=g_{0}(t), u(1, t)=g_{1}(t)$. 
It is immediate from these definitions that in the saturated set $\mathcal{S}(u)=$ $\{u \leq 0\}$, we have the representation

$$
u(x, t)=\frac{g_{1}(t)}{1-s(t)}(x-s(t))
$$

while the following conditions hold on $x=s(t)$ if $s(t)<1$

$$
\begin{aligned}
& u(s(t), t)=0, t \in(0, T), \\
& \frac{\partial u}{\partial x}(s(t), t)=\frac{g_{1}(t)}{1-s(t)}, t \in(0, T) .
\end{aligned}
$$

For a general solution, the free boundary $\Gamma(u)$ is defined as the boundary of the positivity set located inside $Q_{T}$ :

$$
\Gamma(u)=\partial \mathcal{P}(u) \cap Q_{T}
$$

From the results of [5], [13] we know that, under Lipschitz continuous assumptions on the data, a weak solution $u$ of problem $(2.1)-(2.4)$ exists and is unique. Moreover, this solution $u$ is a classical free-boundary solution as in Definition (2.2).

Further regularity of the solution can be proved assuming that the constitutive function $c(\eta)$ satisfies a smoothness condition at $u=0, c^{\prime}(0)=0$ (see [5]). Moreover, from the results of [13], a comparison principle for weak solutions of problem (PD) holds in the following sense:

Comparison. Let $u_{1}$ and $u_{2}$ be the weak solutions of (2.1)-(2.4) corresponding to the initial data $v_{01}$ and $v_{02}$ and boundary data $u_{j}(i, t)=g_{i j}(t), i=0,1, j=1,2$. If the data are ordered, i.e., $v_{01}(x) \geq v_{02}(x)$ in $(0,1)$, and $u_{1}(i, t) \geq u_{2}(i, t)$ for $i=0,1,0 \leq t \leq T$, then $c\left(u_{1}\right) \geq c\left(u_{2}\right)$ on $Q_{T}$.

From the Comparison Principle stated above, if $c\left(u_{1}\right) \geq c\left(u_{2}\right)$ we get the conclusion $\mathcal{P}\left(u_{2}\right) \subseteq \mathcal{P}\left(u_{1}\right)$. In $\mathcal{P}\left(u_{2}\right)$ we have $u_{1} \geq u_{2}$. In $\mathcal{N}\left(u_{2}\right)$, the inequality $u_{1} \geq u_{2}$ follows from the linear expression (2.7) of the solution.

The definition of classical free boundary solution can be extended to suband super-solutions as follows:

Classical free boundary sub- and super-solutions. For the classical free boundary sub-solution, Definition 2.1 is changed as follows: the equation to be satisfied by $u$ on both $P(u)$ and $N(u)$ is replaced by an inequality $\leq$. Point (iii) becomes: $u_{x}$ may stay continuous over the free boundary or admit jumps upwards, $u_{x}\left(s(t)^{-}, t\right) \leq u_{x}\left(s(t)^{+}, t\right)$. Note that $u(x, t)$ is still a continuous function in the whole domain, $u \in C^{0}\left(Q_{T}\right)$.

For the classical free boundary super-solution, the inequalities are reversed.

In Section 2.2 we will show some examples of classical free boundary sub and supersolution for problem (2.1)-(2.4). 


\subsection{Travelling wave examples}

Next, we find some examples of classical free boundary solutions and sub- or supersolutions. The most popular one is the family of travelling waves: we look for solutions of problem (2.1)-(2.4) of the type

$$
u(x, t)=U\left(x-x_{0}-\lambda t\right)=U(\eta), \quad \lambda \in \mathbf{R} .
$$

The curve $\{\eta=0\}$ separates the region $\{\eta<0\}$ where $U>0$ from the region $\{\eta>0\}$ where $U<0$. The line $x=x_{0}+\lambda t$ is the free boundary.

In the region $\{U>0\}$ the solution $U$ of equation (2.1) satisfies:

$$
\begin{aligned}
& -\lambda c(U)^{\prime}(\eta)-U^{\prime \prime}(\eta)=0, \text { if } \eta<0 \\
& U(0)=0, \\
& U^{\prime}(0)=-K,
\end{aligned}
$$

where $K>0$. We remark that the constant $\lambda$ can be negative (then the free boundary will be decreasing in $t$ ). Integrating with respect to $\eta$, taking into account (2.12) and (2.13):

$$
\begin{aligned}
& -\lambda c(U(\eta))-U^{\prime}(\eta)=K, \quad \text { for } \eta \leq 0, \\
& U(0)=0 .
\end{aligned}
$$

The Cauchy problem (2.14) has a unique solution $U(\eta)$. If we define

$$
F(U): \equiv \int_{0}^{U} \frac{d U}{K+\lambda c(U)}, \quad U>0,
$$

then (2.14) is equivalent to

$$
F(U)=-\eta
$$

Note that $F$ depends on the speed parameter $\lambda$. If $\lambda \geq 0$ function $F(U)$ is invertible for $U>0$, since

$$
F^{\prime}(U)=\frac{1}{K+\lambda c(U)}>0 .
$$

On the other hand, if $\lambda<0$, we get an orbit $U(\xi)$ starting from $U(0)=0$, such that

$$
0 \leq U<c^{-1}\left(-\frac{K}{\lambda}\right) .
$$

In this range $F(U)$ is invertible. Hence, we obtain $U=F^{-1}\left(x_{0}+\lambda t-x\right)$.

In the particular case of linear $c: c(u)=u$ for $u>0$, simple calculations give explicit formulas. We obtain that $U\left(x-x_{0}-\lambda t\right)$ has the following form:

$$
\begin{aligned}
& U\left(x-x_{0}-\lambda t\right)=\frac{K}{\lambda}\left(\exp \left(-\lambda\left(x-x_{0}-\lambda t\right)\right)-1\right), \quad \text { if } x<x_{0}+\lambda t, \\
& U\left(x-x_{0}-\lambda t\right)=-K\left(x-x_{0}-\lambda t\right), \quad \text { if } x \geq x_{0}+\lambda t .
\end{aligned}
$$


We can construct classical free boundary sub- and supersolution of problem (2.1)-(2.4), taking $U$ satisfying equation (2.11)-(2.13) with suitable constraints on $x_{0}$ and on $K$ (for example $K \geq\left|g_{1}(t)\right| /\left(1-x_{0}-\lambda t\right)$ ) and replacing the equality in (2.11) respectively by $\leq 0$ or $\geq 0$.

The only constraint on $\lambda$ is: $\lambda<\frac{1-x_{0}}{T}$, if $\lambda>0$ and $\lambda>-\frac{x_{0}}{T}$, if $\lambda<0$.

In a similar way we can write explicit classical free boundary sup- and supersolutions for the Neumann problem.

Remark 2.1 Note that, by adjusting the free parameters $K$, slope at the free boundary, and $\lambda$, wave speed, any classical free boundary solution can be locally approximated in $C^{1}$ norm by a travelling wave solution. A corresponding statement can be formulated for super- and subsolutions.

In the following section we give the definition of viscosity solution by means of the definition of classical free boundary super- and sub solutions given above. In the subsequent Sections 4 and 5 we prove the well-posedness in this viscosity setting for Lipschitz continuous data, and finally for merely continuous data.

\section{$3 \quad$ Viscosity solutions}

Following the standard approach, we introduce first the definitions of super- and sub-solution. Let $Q_{T}=(0,1) \times(0, T)$.

Definition 3.1 A function $u(x, t) \in C^{0}\left(Q_{T}\right)$ is a viscosity subsolution of equation (2.1) if:

(i) At every point $P_{0}=\left(x_{0}, t_{0}\right)$ in $\mathcal{P}(u) \cap Q_{T}$ and for every $\varphi \in C^{2,1}\left(Q_{T}\right)$ that touches $u$ from above at point $P_{0}$, the inequality

$$
\frac{\partial c(\varphi)}{\partial t}-\frac{\partial^{2} \varphi}{\partial x^{2}} \leq 0
$$

holds in $P_{0}$.

(ii) At every point $P_{0}=\left(x_{0}, t_{0}\right)$ in $\mathcal{N}(u) \cap Q_{T}$ and for every function $\varphi \in C^{2,1}\left(Q_{T}\right)$ that touches $u$ from above at point $P_{0}$, the inequality

$$
-\frac{\partial^{2} \varphi}{\partial x^{2}} \leq 0
$$

holds in $P_{0}$.

(iii) Let $P_{0}=\left(x_{0}, t_{0}\right) \in \partial \mathcal{P}(u) \cap Q_{T}$ and let $\mathcal{R}$ be a parabolic neighborhood of $P_{0}, \mathcal{R}=\left(x_{0}-\delta_{1}, x_{0}+\delta_{1}\right) \times\left(t_{0}-\delta_{2}, t_{0}\right)$, with $\delta_{1}$ and $\delta_{2}>0$ and $\mathcal{R} \subset Q_{T}$. Then, for every classical free-boundary supersolution $U_{+}$, if $u<U_{+}$on the parabolic boundary of $\mathcal{R}$ it follows that $u \leq U_{+}$on $\mathcal{R}$.

A function $u(x, t) \in C^{0}([0,1] \times[0, T))$ is a viscosity subsolution of problem (2.1)-(2.4) if it is a viscosity solution of equation (2.1) and the initial and boundary 
data are well adjusted:

(iv) $c(u(x, 0)) \leq v_{0}(x), \forall x \in(0,1) ; u(i, t) \leq g_{i}(t), i=0,1, t \in(0, T)$.

A similar definition applies to viscosity supersolutions after changing the sign of the inequalities involved in the definition in the obvious way.

Definition 3.2 A viscosity solution of problem (2.1)-(2.4) is a continuous function defined in $Q_{T}$ which is at the same time a sub- and a super-solution.

Any classical free boundary sub- (resp. supersolution) can be locally approximated by a sub- (resp. supersolution) of the type "travelling wave" found in the previous section (see Remark 2.1). Hence, in the definition of viscosity solution we can replace any classical free boundary sub- (resp. supersolution) by a travelling wave sub- (resp. supersolution). In this way we restrict the class of functions to test the comparison principle in the neighborhoods of the points of $\partial \mathcal{P}(u) \cap Q_{T}$ to a very special explicit family.

\section{Well posedness for Lipschitz continuous data}

As a preliminary for our general well-posedness result, we prove that, in the case of Lipschitz continuous data, the viscosity solution exists and coincides with the classical free boundary solution.

Theorem 4.1 Under the assumptions

(i) $g_{i}(t), i=0,1$ are Lipschitz continuous functions on $[0, T]$,

(ii) $g_{0}(t)>0$ for all $t \in(0, T)$, and $g_{1}(t)<0$ for all $t \in(0, T)$,

(iii) $v_{0}(x) \geq 0$, and there exists a Lipschitz continuous function $u_{0}:(0,1) \rightarrow \mathbf{R}$

such that $v_{0}=c\left(u_{0}\right)$,

(iv) $u_{0}(x)>0$ iff $x \in\left[0, x_{0}\right), x_{0} \in(0,1)$,

(iv) $u_{0}(0)=g_{0}(0), u_{0}(1)=g_{1}(1)$,

the initial boundary value problem (2.1)-(2.4) is well posed in the class of viscosity solutions: the viscosity solution coincides with the continuous weak solution of the problem.

Proof. Let $w$ be the continuous weak solution of the problem (2.1)-(2.4). Under the above assumptions, we know from the results of [5], [13] that $w$ exists, it is unique and is a classical free boundary solution.

Viscosity SOlution. Existence. We now prove that such a $w$ is a viscosity solution. First we prove that $w$ is a viscosity subsolution. The argument is quite standard. Let $\varphi \in C^{2,1}\left(Q_{T}\right)$ be a test function that touches $w$ from above in $P_{0}=\left(x_{0}, t_{0}\right) \in Q_{T}$, where $w\left(P_{0}\right)>0$. In $P_{0}$ the function $\varphi-w$ has a minimum zero, then at this point the following is true:

$$
\varphi=w, \varphi_{t} \leq w_{t}, \varphi_{x x} \geq w_{x x} .
$$


Since $w$ is a classical solution in $\mathcal{P}(w)$, it satisfies $c(w)_{t}-w_{x x}=0$ in $P_{0}$. Hence,

$$
c(\varphi)_{t}-\varphi_{x x} \leq 0, \text { in } P_{0} .
$$

If $w\left(P_{0}\right)<0$, we analogously obtain

$$
-\varphi_{x x} \leq 0 \text {, in } P_{0}
$$

If $w\left(P_{0}\right)=0$, let $I$ be a parabolic neighbourhood of $P_{0}$ and let $U_{+}$be a classical free boundary supersolution such that $w<U_{+}$on the parabolic boundary of $I$. A comparison principle between weak solutions holds ([13]), then we conclude that $w \leq U_{+}$on $I$.

Hence, we have proved that $w$ is a viscosity subsolution. In a similar way, we prove that $w$ is a viscosity supersolution. Besides, every classical free boundary solution is a viscosity solution (consistency).

UNIQUENESS. We want to prove that if $u$ is another viscosity solution to problem (2.1)-(2.4), then $u=w$. We will prove that $u \leq w$ and then that $u \geq w$.

A) Proof that $u \leq w$. Under the assumptions of the theorem, we know that a Lipschitz function $u_{0}$ exists such that $c\left(u_{0}\right)=v_{0}$. Let us consider $w_{\epsilon}$ the weak solution of

$$
\begin{array}{ll}
c\left(w_{\epsilon}\right)_{t}-w_{\epsilon x x}=0, & \text { in } Q_{T}, \\
c\left(w_{\epsilon}\right)(x, 0)=c\left(u_{0}(x)+\epsilon\right), & \text { on }(0,1), \\
w_{\epsilon}(0, t)=g_{0}(x, t)+\epsilon, & \text { on }(0, T), \\
w_{\epsilon}(1, t)=g_{1}(x, t)+\epsilon, & \text { in }(0, T),
\end{array}
$$

The constant $\epsilon>0$ is taken sufficiently small such that $g_{1}(x, t)+\epsilon<0$ and such that $u_{0}(x)+\epsilon>0$, for $x \in\left[0, x_{\epsilon}\right)$ and $u_{0}(x)+\epsilon \leq 0$, for $x \in\left[x_{\epsilon}, 1\right]$. We know from [5] that there exists an unique weak solution $w_{\epsilon}$ of problem (4.1). This is a classical free boundary supersolution of problem (2.1)-(2.4).

From the comparison principle between weak solutions ([13]) we know that: if $w$ and $w_{\epsilon}$ are solution respectively of problems (2.1)-(2.4) and (4.1), then $c(w) \leq$ $c\left(w_{\epsilon}\right)$. As shown in Section 2.1, this implies that also $w \leq w_{\epsilon}$ in $Q_{T}$.

Moreover, we have that $w_{\epsilon}$ is a nondecreasing sequence of $\epsilon$, hence there exists the limit as $\epsilon$ tends to 0 and from the uniqueness of the weak solution, this limit is $w$ :

$$
\lim _{\epsilon \rightarrow 0} w_{\epsilon}=w .
$$

We want to prove that for any $u(x, t)$, viscosity solution of problem (2.1)-(2.4), we have $u(x, t)<w_{\epsilon}(x, t)$, in $Q_{T}, \forall \epsilon>0$, sufficiently small. Suppose for contradiction that there exists a first point $\bar{P}=(\bar{x}, \bar{t}) \in Q_{T}$ such that $u(\bar{x}, \bar{t})=w_{\epsilon}(\bar{x}, \bar{t})$. We examine the different possibilities.

(i) The point $\bar{P}=(\bar{x}, \bar{t})$ lies in the positivity set $\mathcal{P}(u)$, so that $u(\bar{x}, \bar{t})=$ $w_{\epsilon}(\bar{x}, \bar{t})>0$. 
Then, there exists a rectangular parabolic neighborhood $B$ of $\bar{P}=(\bar{x}, \bar{t})$, such that $u(x, t)>0$ and $w_{\epsilon}(x, t)>0, \forall(x, t) \in B$ and such that $u<w_{\epsilon}$ on its parabolic boundary. The function $w_{\epsilon}$ is smooth in its positivity set and it solves equation $c^{\prime}\left(w_{\epsilon}\right) w_{\epsilon t}-w_{\epsilon x x}=0$ in the classical sense in $B$.

Now we can find a classical solution $\tilde{u}$ such that $\tilde{u}=u+\gamma$ on $\partial_{p} B$, with $\gamma>0$ such that $\tilde{u}<w_{\epsilon}$ on $\partial_{p} B$. From the Strong Maximum Principle between classical solutions we have that $\tilde{u}(\bar{P})<w_{\epsilon}(\bar{P})$. We prove that $u \leq \tilde{u}$ in $B$ : we approximate $\tilde{u}$ by $\tilde{u}_{\delta}, \delta>0$, classical solution in $B$ of

$$
c^{\prime}\left(\tilde{u}_{\delta}\right) \tilde{u}_{\delta t}-\tilde{u}_{\delta x x}=\delta
$$

with boundary data $\tilde{u}_{\delta}=\tilde{u}+\delta$. If $\tilde{u}_{\delta}$ touches from above $u$ at a point $P_{1}$ of $B$, from the definition of viscosity subsolution and the smoothness of $\tilde{u}_{\delta}$, we have $c^{\prime}\left(\tilde{u}_{\delta}\right) \tilde{u}_{\delta t}-\tilde{u}_{\delta x x} \leq 0$ which is a contradiction. Hence, $u<\tilde{u}_{\delta}$ in $B$. Since $\tilde{u}_{\delta} \searrow \tilde{u}$, in the limit $u \leq \tilde{u}$ in $B$. Hence, $u \leq \tilde{u}(\bar{P})<w_{\epsilon}(\bar{P})$, thus a contradiction.

(ii) $\bar{P}=(\bar{x}, \bar{t}) \in \mathcal{N}(u)$. Then, we have $u(\bar{x}, \bar{t})=w_{\epsilon}(\bar{x}, \bar{t})<0$. There exists a neighborhood of $\bar{P}, B=\left(x_{1}, x_{2}\right) \times\left(t_{1}, \bar{t}\right)$, such that $u<0, w_{\epsilon}<0$ in $B$ and $u<w_{\epsilon}$ on its parabolic boundary. Since $w_{\epsilon}$ is smooth in $\mathrm{B}$, we have that $w_{\epsilon x x}(x, t)=0$ in $B$.

Consider now $w_{\epsilon \delta}=w_{\epsilon}-\delta\left(x-x_{1}\right)^{2}$, with $\delta>0$ suitably small such that $w_{\epsilon \delta}\left(x_{2}, t\right)>u\left(x_{2}, t\right)$. If, in a point $P_{0} \in \bar{B}, w_{\epsilon \delta}$ touches $u$ from above, from the definition of viscosity solution we have that $w_{\epsilon \delta x x} \geq 0$ in $P_{0}$, on the other hand $w_{\epsilon \delta x x}=-2 \delta<0$, hence in $\bar{B}$ we have $u<w_{\epsilon \delta} \leq w_{\epsilon}$, i.e. a contradiction.

(iii) Finally, we examine the interesting case when contact takes place at the free boundary: $\bar{P}=(\bar{x}, \bar{t}) \in \Gamma(u): \equiv \partial \mathcal{P}(u) \cap Q_{T}$. Then $(\bar{x}, \bar{t})$ belongs to the free boundary $x=s_{\epsilon}(t)$, which is the curve where $w_{\epsilon}=0$ and $u(\bar{x}, \bar{t})=w_{\epsilon}(\bar{x}, \bar{t})=0$.

Note that the curve $x=s_{\epsilon}(t)$ can not touch the axes $x=0,1$ since $g_{0}+\epsilon>0$ and $g_{1}+\epsilon<0$, hence $\bar{x}=s_{\epsilon}(\bar{t})<1$.

We take a rectangular parabolic neighborhood $B$ of $\bar{P}=(\bar{x}, \bar{t})$ such that $u<w_{\epsilon}$ on its parabolic boundary. We may also assume that contact does not take place at other points at time $\bar{t}$, hence there is a positive constant $\gamma=\gamma(\epsilon)$ such that

$$
u \leq w_{\epsilon}-\gamma(\epsilon) \text { on } \partial_{p} B
$$

We now define $w_{\epsilon \delta}$ as the translation of $w_{\epsilon}$ to the left

$$
w_{\epsilon \delta}(x, t)=w_{\epsilon}(x+\delta, t)
$$

where $\delta$ is taken small enough so that we still have

$$
u(x, t)<w_{\epsilon \delta}(x, t)
$$

on the parabolic boundary $\partial_{p} B$. From the definition of viscosity solution, it follows that $u \leq w_{\epsilon \delta}$ in $\bar{B}$. But this implies that $u$ must be negative in the set of points of $B$ where $x>s_{\epsilon}(t)-\delta$. In the limit $t=\bar{t}$ we get $u(x, \bar{t})<0$ for $x>s_{\epsilon}(\bar{t})-\delta$, a contradiction with the assumption that $u$ and $w_{\epsilon}$ touch at $t=\bar{t}, x=s_{\epsilon}(\bar{t})$. 
We have proved that $u<w_{\epsilon}$ in $Q_{T}$. Passing to the limit as $\epsilon$ tends to zero, this implies that $u \leq w$ in $Q_{T}$.

B) To prove that $u \geq w$, we consider the function $u_{-\epsilon}$ obtained by solving the problem with initial boundary conditions $w_{-\epsilon}(x, 0)=c\left(u_{0}(x)-\epsilon\right), u_{-\epsilon}(i, t)=$ $g_{i}(t)-\epsilon, i=0,1$ and, by a similar technique as above, we prove that $w_{-\epsilon}<u$, in $Q_{T}$, thus obtaining $u \geq w$ in $Q_{T}$.

\section{$5 \quad$ Well-posedness for general data}

The aim of this section is to prove a well-posedness result for the problem with initial and boundary data which are merely continuous.

The viscosity solution $u$ is constructed as the limit of viscosity solutions whose data are Lipschitz continuous functions and whose existence has been proved in the previous section.

Theorem 5.1 Under assumptions (2.5), (2.6), the initial boundary value problem (2.1)-(2.4) is well posed in the class of viscosity solutions and the viscosity solution coincides with the continuous weak solution of the problem.

Proof. Let us consider the sequences $\left\{\bar{g}_{0 n}(t)\right\},\left\{\underline{g}_{0 n}(t)\right\},\left\{\bar{g}_{1 n}(t)\right\},\left\{\underline{g}_{1 n}(t)\right\}$, $\left\{\bar{u}_{0 n}(x)\right\},\left\{\underline{u}_{0 n}(x)\right\}$ such that:

$$
\begin{aligned}
& \bar{g}_{0 n}(t), \underline{g}_{0 n}(t)>0, \bar{g}_{1 n}(t), \underline{g}_{1 n}(t)<0, \forall n \in \mathbf{N}, \forall t \in(0, T), \\
& \underline{u}_{0 n}(x)>0, \text { iff } x \in\left[0, \underline{x}_{0 n}\right), \underline{x}_{0 n} \in\left(x_{0}, 1\right), \\
& \bar{u}_{0 n}(x)>0, \text { iff } x \in\left[0, \bar{x}_{0 n}\right), \underline{x}_{0 n} \in\left(0, x_{0}\right), \\
& \bar{g}_{i n}, \underline{g}_{i n}, i=0,1, \bar{u}_{0 n}, \underline{u}_{0 n} \text { are Lipschitz continuous functions, } \forall n \in \mathbf{N}, \\
& \left\{\bar{g}_{i n}\right\},\left\{\bar{u}_{0 n}\right\} \text { are decreasing sequences of functions such that } \\
& \bar{g}_{i n} \searrow g_{0}, i=0,1, \bar{u}_{0 n} \searrow u_{0} \text { in } C^{0} \text {, as } n \rightarrow+\infty, \\
& \left\{\underline{g}_{i n}\right\},\left\{\underline{u}_{0 n}\right\} \text { are increasing sequences of functions such that } \\
& \underline{g}_{i n} \nearrow g_{i}, i=0,1, \underline{u}_{0 n} \nearrow u_{0}, \text { in } C^{0}, \text { as } n \rightarrow+\infty .
\end{aligned}
$$

From Theorem 4.1 we know that problem (2.1)-(2.4) with initial and boundary data $\bar{g}_{0 n}, \bar{g}_{1 n}, \bar{u}_{0 n}$ has a unique viscosity solution $\bar{u}_{n}$ which coincides with the classical free boundary solution. Analogously, we denote by $\underline{u}_{n}$ the viscosity solution corresponding to the data $\underline{g}_{0 n}(t), \underline{g}_{1 n}(t), \underline{u}_{0 n}$.

From the comparison principle between classical free boundary solutions we have that $\left\{\bar{u}_{n}\right\}$ and $\left\{\underline{u}_{n}\right\}$ are respectively decreasing and increasing sequences. Moreover $\underline{u}_{n} \leq \bar{u}_{n}$, for all $n \in \mathbf{N}$.

From the monotonicity of the sequences, we have that there exist the following two limits:

$$
\lim _{n} \bar{u}_{n} \equiv: \bar{u}, \quad \lim _{n} \underline{u}_{n} \equiv: \underline{u} .
$$


From the continuity and the monotonicity of $\bar{u}_{n}$ and $\underline{u}_{n}$ we have that $\bar{u}$ is an upper semicontinuous function and $\underline{u}$ is a lower semicontinuous function.

Continuity. We will prove that $\bar{u}=\underline{u}$ by means of a continuous dependence result for the functions $\bar{u}_{n}, \underline{u}_{n}$.

Let $\bar{v}_{n}$ and $\underline{v}_{n}$ be such that $c\left(\bar{u}_{n}\right)=\bar{v}_{n}$ and $c\left(\underline{u}_{n}\right)=\underline{v}_{n}$. Denote by $v_{n}:=$ $\bar{v}_{n}-\underline{v}_{n} \geq 0, u_{n}:=\bar{u}_{n}-\underline{u}_{n} \geq 0$. The functions $v_{n}, u_{n}$ solve the following problem

$$
\left\{\begin{array}{l}
\frac{\partial v_{n}}{\partial t}-\frac{\partial^{2} u_{n}}{\partial x^{2}}=0, \text { in }(0,1) \times(0, T) \\
v_{n}(x, 0)=c\left(\bar{u}_{0 n}(x)\right)-c\left(\underline{u}_{0 n}(x)\right)=: v_{0 n}(x)>0, \text { on }(0,1), \\
u(0, t)=\bar{g}_{0 n}(t)-\underline{g}_{0 n}(t)=: g_{0 n}(t)>0, \text { on }(0, T), \\
u(1, t)=\bar{g}_{1 n}(t)-\underline{g}_{1 n}(t)=: g_{1 n}(t)>0, \text { on }(0, T) .
\end{array}\right.
$$

We now take a test function $\varphi(x) \in C^{2}([0,1])$ with the following properties:

$$
\varphi(0)=\varphi(1)=0, \frac{\partial^{2} \varphi}{\partial x^{2}}=-1 .
$$

Under assumptions (5.4), we have $|\partial \varphi / \partial x| \leq C$. Multiplying equation (5.3) by $\varphi(x)$ and integrating in $(0,1) \times(0, t)$, we obtain

$$
\begin{aligned}
& \int_{0}^{1} v_{n} \varphi d x-\int_{0}^{1} v_{0 n} \varphi d x=\int_{0}^{t} u_{n x} \varphi /{ }_{0}^{1} d t-\int_{0}^{t} u_{n} \varphi_{x} /{ }_{0}^{1} d t \\
& +\int_{0}^{t} \int_{0}^{1} u_{n} \varphi_{x x} d x d t .
\end{aligned}
$$

Taking into account (5.4), we obtain

$$
\begin{aligned}
& \int_{0}^{1} v_{n}(x, t) \varphi(x) d x+\int_{0}^{t} \int_{0}^{1} u_{n}(x, t) d x d t \leq \int_{0}^{1} v_{0 n}(x) \varphi(x) d x \\
& +C \int_{0}^{t}\left(g_{0 n}(t)+g_{1 n}(t)\right) d t
\end{aligned}
$$

If we choose the data such that

$$
\int_{0}^{t}\left(g_{0 n}+g_{1 n}\right) d t \leq \epsilon, \int_{0}^{1} v_{0 n} d x \leq \epsilon,
$$

from (5.6), we obtain that

$$
\int_{0}^{t} \int_{0}^{1} u_{n}(x, t) d x d t \leq 2 \epsilon,
$$

and, from the nonnegativity of $u_{n}$, we obtain that $u_{n}=\bar{u}_{n}-\underline{u}_{n} \rightarrow 0$ as $n \rightarrow+\infty$. Hence $\bar{u}=\underline{u}=: u$ almost everywhere. 
Now we prove that $\bar{u}=\underline{u}=: u$ for all $(x, t) \in Q_{T}$. Where $\bar{u}$ and $\underline{u}$ are both smooth functions, there the equality $\bar{u}=\underline{u}$ holds and also $\bar{v}=c(\bar{u}) \equiv \underline{v}=c(\underline{u})$. If we prove that the corresponding free boundaries coincide, then we can conclude that $\bar{u} \equiv \underline{u}=: u$ for all $(x, t) \in Q_{T}$.

Let $\left\{\bar{s}_{n}(t)\right\},\left\{\underline{s}_{n}(t)\right\}$ be the sequences of the free boundaries related to $\left\{\bar{u}_{n}\right\}$, $\left\{\underline{u}_{n}\right\}$. Clearly $\left\{\bar{s}_{n}(t)\right\}$ is a decreasing sequence and $\left\{\underline{s}_{n}(t)\right\}$ is increasing. Define

$$
\lim _{n} \bar{s}_{n} \equiv: \bar{s}, \quad \lim _{n} \underline{s}_{n} \equiv: \underline{s} .
$$

From the continuity and the monotonicity of $\bar{s}_{n}$ and $\underline{s}_{n}$ we have that $\bar{s}$ is an upper semicontinuous function and $\underline{s}$ is a lower semicontinuous function, $\bar{s}_{n} \geq \underline{s}_{n}$ for all $n \in \mathbf{N}$.

Suppose that there exists a point $t^{*}$ such that $\bar{s}_{n}\left(t^{*}\right)>\underline{s}_{n}\left(t^{*}\right)$, then integrating on $(0,1)$ for $t=t^{*}$ we have

$$
\int_{0}^{1} \bar{v}\left(x, t^{*}\right) d x>\int_{0}^{1} \underline{v}\left(x, t^{*}\right) d x,
$$

which is a contradiction with the integral inequality (5.6) if we choose $n$ sufficiently large.

Hence $\bar{s}(t)=\underline{s}(t) \equiv: s(t)$ for all $t \in[0, T]$ and $s(t)$ is a continuous function. This implies that $\bar{u}=\underline{u}=: u$ for all $(x, t) \in Q_{T}$.

Hence, we have proved that the limit function $u$ obtained as lower limit of decreasing functions or as upper limit of increasing functions is continuous.

Let us point out that the continuity of $u, \bar{u}_{n}, \underline{u}_{n}$ and the monotonicity of the sequences $\left\{\bar{u}_{n}\right\},\left\{\underline{u}_{n}\right\}$ implies that the convergences in (5.2) are locally uniform.

The Limit $u$ is A Viscosity solution. We prove that the common limit $u$ is a viscosity solution to problem (2.1)-(2.4) with initial and boundary data satisfying assumptions (2.5), (2.6).

We first prove that $u$ is a viscosity subsolution.

1) $P_{0}=\left(x_{0}, t_{0}\right)$ is a point such that $u\left(P_{0}\right)>0$ or $u\left(P_{0}\right)<0$ :

in this case the procedure is standard, by using the definition of viscosity solution for the functions $\bar{u}_{n}$ and using that the sequence $\left\{\bar{u}_{n}\right\}$ converges uniformly to $u$ (see, for example, Proposition 2.2 of [3]).

2) $P_{0}=\left(x_{0}, t_{0}\right)$ is a point such that $u\left(P_{0}\right)=0$ : we have to verify that every classical free-boundary supersolution $U_{+}$such that $u<U_{+}$on the parabolic boundary of an arbitrary neighbourhood $I$ of $P_{0}$, then $u \leq U_{+}$on $I$.

Since $\bar{u}_{n}$ converges uniformly to $u$, we choose an $n$ sufficiently large such that $\bar{u}_{n}<U_{+}$on the parabolic boundary of $I$. Since $\bar{u}_{n}$ is a viscosity solution we know that $\bar{u}_{n} \leq U_{+}$on $I$ and then $u<\bar{u}_{n} \leq U_{+}$.

We proceed analogously to prove that $u$ is a viscosity supersolution.

UNIQUENESS. Let $\tilde{u}$ be an other viscosity solution, we prove that $\tilde{u}=u$. In Theorem 4.1 we have proved that the viscosity solutions $\bar{u}_{n}$ and $\underline{u}_{n}$ coincide 
with the classical free-boundary solutions corresponding to the same data. By construction we have that $\tilde{u}<\bar{u}_{n}$, for all $n \in \mathbf{N}$, on the parabolic boundary of $Q_{T}$. Hence, from the comparison between viscosity solutions and classical freeboundary solutions (see the proof of Theorem 5.1) we have that $\tilde{u}<\bar{u}_{n}$, for all $n \in \mathbf{N}$, hence passing to the limit as $n \rightarrow+\infty, \tilde{u} \leq u$. Analogously $\tilde{u}>\underline{u}_{n}$ for all $n \in \mathbf{N}$, hence $\tilde{u} \geq \underline{u}$.

Continuous Dependence For General Data. Passing to the limit as $n \rightarrow+\infty$ we have that the equality (5.5) holds true also for the viscosity solution $u$,

$$
\int_{0}^{1} c(u) \varphi d x-\int_{0}^{t} \int_{0}^{1} u \varphi_{x x} d x d t=\int_{0}^{1} v_{0} \varphi d x-\int_{0}^{t} u \varphi_{x} /_{0}^{1} d t
$$

for all $\varphi(x) \in C^{2,1}$ such that $\varphi(0)=\varphi(1)=0$. Hence, $u$ is also a weak solution in the sense specified in $([5]),([13])$.

We have also found that the free boundary is a continuous function $x=s(t)$. Moreover, from the uniqueness results of F. Otto ([16]) of the weak solution of problem (2.1)-(2.4), we obtain that the unique viscosity solution $u$ of problem (2.1)-(2.4) with continuous data coincides with the unique weak solution of this problem.

\section{Extensions and comments}

Problem in several dimensions. We want to emphasize that Theorem 5.1 can be proved only in the one dimensional case since we strongly use the results on existence, uniqueness, regularity, comparison principle for weak solutions by [13] and [5] which are true only in this case. As shown by the work on the problem of the heat equation with combustion-type boundary conditions, [9], [15], uniqueness of viscosity solutions in these free boundary problems in not easy in space several dimensions.

Neumann and mixed problems. Similar results can be proved for the well posedness of the Neumann problem $(\mathrm{PN})$, obtained by replacing conditions $(2.3)$ and (2.4) by

$$
u_{x}(0, t)=f_{0}(t), \quad u_{x}(1, t)=f_{1}(t) .
$$

In this case the Definition (2.2) of classical free boundary solution is naturally modified in conditions $(\mathrm{v})$. We recall that classical results on the existence and uniqueness of a weak solution with Neumann boundary conditions can be still found in [13] and [5].

For the Neumann problem $(2.1),(2.2),(6.1)$ we can give a similar definition of viscosity subsolution replacing condition iv) in Definition (3.1) by

$$
c(u(x, 0)) \leq v_{0}(x), u_{x}(0, t) \geq f_{0}(t), u_{x}(1, t) \leq f_{1}(t),
$$

and in Definition of supersolution by (6.2) with reversed inequalities. 
Since the techniques used above are local, we can use the same procedure to prove the existence and uniqueness of a viscosity solution which coincides with the weak solution.

Analogous results can be obtained by considering mixed boundary conditions.

\section{Acknowledgements}

Work partially supported by the Universidad Autónoma de Madrid, the Italian M.I.U.R. project "Viscosity, metric, and control theoretic methods for nonlinear partial differential equations" and by Spanish MCYT Project BMF2002-04572C02-02.

\section{References}

[1] H. W. ALT and S. LUCKHAUS, Quasilinear Elliptic-Parabolic Differential Equations, Math. Z. 183(1) (1983), 311-341.

[2] I. ATHANASOPOULOS, L. CAFFARELLI and S. SALSA, Regularity of the free boundary in parabolic phase-transition problems, Acta Math. 176(2) (1996), 245-282.

[3] M. BARDI and I. CAPUZZO DOLCETTA, Optimal control and viscosity solutions of Hamilton-Jacobi Bellman equations, Systems and Control: Foundations and Applications, Birkhauser, Boston, MA, 1997.

[4] G. BARLES, Solutions de viscosite des equations de Hamilton-Jacobi, Mathematiques and applications 17, Springer Verlag, Paris, 1994.

[5] M. BERTSCH and J. HULSHOF, Regularity results for an elliptic-parabolic free boundary problem, Trans. Amer. Math. Soc. 297(1) (1986), 337-350.

[6] C. BRANDLE and J. L. VAZQUEZ, Viscosity solutions for quasilinear parabolic equations, Preprint, 2003.

[7] L. CAFFARELLI, A Harnack inequality approach to the regularity of free boundaries. I. Lipschitz free boundaries are $C^{1, \alpha}$. Rev. Mat. Iberoamericana 3(2) (1987), 139-162.

[8] L. CAFFARELLI and X. CABRÈ, Fully nonlinear elliptic equation, Coll. Publ 43, Amer. Math. Soc., Providence, 1995.

[9] L. A. CAFFARELLI, C. LEDERMAN and N. WOLANSKI, Pointwise and viscosity solutions for the limit of a two phase parabolic singular perturbation problem. Indiana Univ. Math. J. 46(3) (1997), 719-740. 
[10] L. CAFFARELLI and J. L. VAZQUEZ, Viscosity solutions for the Porous medium Equation, Proc. Sympos. Pure Math. 65, Amer. Math. Soc., Providence, 1999, 13-26.

[11] M. G. CRANDALL, H. ISHII and P. L. LIONS, User's guide to viscosity solutions of second-order partial differential equations, Bull. Amer. Math. Soc. 27(1) (1992), 1-67.

[12] E. DI BENEDETTO and R. GARIEPY, Local Behavior of solutions of an elliptic-parabolic equation, Archive Rational Mech. Anal. 1 (1987), 1-17.

[13] C. J. VAN DUIJN and L. A. PELETIER, Nonstationary filtration in partially saturated porous media, Arch. Rational Mech. Anal. 78(2) (1982), 173-198.

[14] A. FASANO and M. PRIMICERIO, Liquid flow in partially saturated porous media, J. Inst. Math. Appl. 23(4) (1979), 503-517.

[15] C. LEDERMAN, J. L. VÁZQUEZ and N. WOLANSKI, Uniqueness of solution to a free boundary problem from combustion, Trans. Amer. Math. Soc. 353(2) (2001), 655-692.

[16] F. OTTO, $L^{1}$-Contraction and Uniqueness for Quasilinear Elliptic-Parabolic Equations, J. Diff. Eq. 131 (1996), 20-38.

Received 16 September 2004; accepted 20 February 2005

To access this journal online:

http://www.birkhauser.ch 